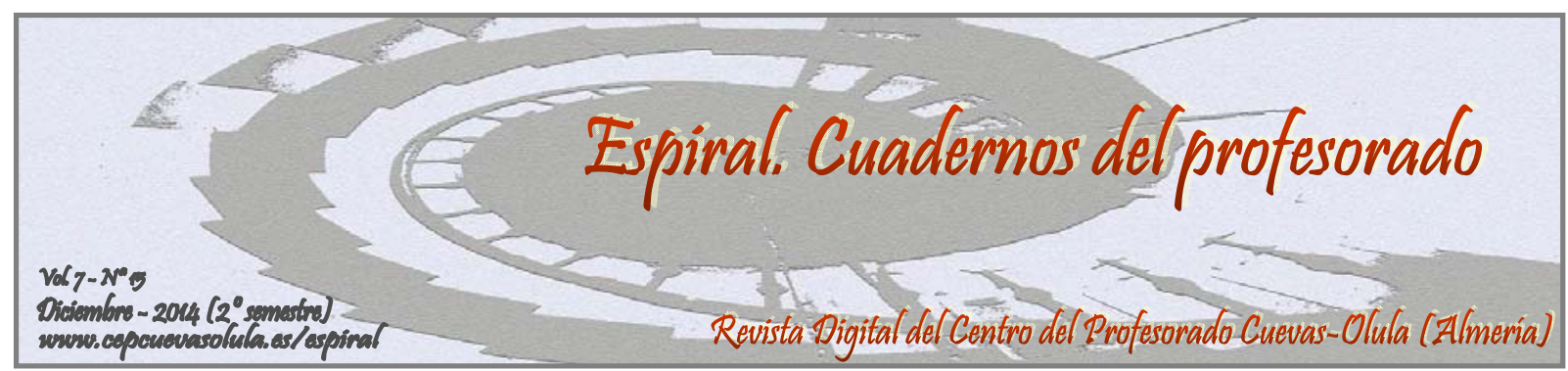

\title{
CONSCIENCIA Y COMPORTAMIENTO AMBIENTALES DE TURISTAS EN EL RIO ARAGUAIA EN LAS VACACIONES DE JULIO
}

\author{
BEHAVIOUR AND ENVIRONMENTAL CONSCIOUSNESS \\ OF TOURISTS IN RIO ARAGUAIA IN JULY HOLIDAY
}

\author{
Humberto Luís de Deus Inácio, Oromar Augusto Do Nascimento, \\ Ana Paula Sales Da Silva y Mateus Jesús Da Silva
}

Facultad de Educación Física, Universidad Federal de Goiás, Brasil

RESUMEN: Este estudio ${ }^{1}$ tuvo como objetivo identificar la relación entre la consciencia ambiental y el comportamiento de practicantes de actividades de ocio en la naturaleza, concretamente de turistas y habitantes locales de las islas del Rio Araguaia durante las vacaciones del mes de julio. Se utilizó la Escala de Comportamiento Responsable, en una muestra de 235 personas. Se partió de la premisa de que la consciencia y el comportamiento no se relacionan de manera lineal. La investigación indicó que el $\mathbf{9 0 . 8 7 \%}$ de los sujetos investigados presentan un nivel de consciencia ambiental adecuado, un número muy cercano de los $90.09 \%$ que conectan este nivel de consciencia con un comportamiento responsable. Los datos indican que existe la necesidad de profundizar en las prácticas pedagógicas, en las acciones y en las políticas públicas que puedan ayudar a las personas a reflexionar sobre su consciencia en sus actitudes.

Palabras clave: Prácticas corporales en la naturaleza, ocio, comportamiento, consciencia ambiental

ABSTRACT: This study aimed identifies the relationship between environmental consciousness and behavior, of practitioners of leisure activities in nature, more specifically frequenters of the islands of the Araguaia River, during vacation in July. Was used a Responsible Behavior Scale with 235 persons. The starting point was the premise that consciousness and behavior or not related linearly, may present distinct relations between behavior and consciousness. The research indicated that $90.87 \%$ had an adequate level of environmental consciousness, very close to the $90.09 \%$ of persons with a adequate level of environmental consciousness. It is assured that there is a need to deepen pedagogical practices that may help people to reflect their consciences in their attitudes.

Key words: Leisure activities in nature practices, leisure, behaviour and environmental conscience

Inácio, H. L. D., Do Nascimento, O. A., Sales Da Silva, A. P., y Da Silva, M. J. (2014). Consciencia y comportamiento ambientales de turistas en el Río Araguaia en las vacaciones de julio. Espiral. Cuadernos del Profesorado, 7(15), 3-7. Disponible en: http://www.cepcuevasolula.es/espiral.

Fecha de recepción: 18/04/2014

Fecha de aceptación: 05/07/2014
Enviar correspondencia a: betoinacio@gmail.com

\footnotetext{
${ }^{1}$ Esta investigación ha tenido apoyo de la UFG - Universidade Federal de Goiás y de la FAPEG - Fundação de Apoio à Pesquisa de Goiás, para su realización.
} 


\section{1.- INTRODUCCIÓN}

Comportamientos y actitudes se reflejan en las formas de pensar, de ver el mundo, del compromiso socio-colectivo de un individuo o un grupo, etc. Del mismo modo, la evaluación de los comportamientos o actitudes se ve influenciada por la perspectiva del espectador, sus objetivos y también los que están siendo evaluados.

Según Corraliza y Martin (2000), a pesar de que está creciendo el interés en el estudio del comportamiento humano en relación con la naturaleza, son pocos los que se dedican a indagar sobre la complejidad de este fenómeno. Rodríguez, Boyes, y Stanisstreet (2010) hacen hincapié en la necesidad de estos estudios, porque afirman que hay una gran diferencia entre la conciencia ambiental de la población y el comportamiento pro-ambiental.

En el caso de esta investigación, se parte del concepto de medio ambiente (MA) como una construcción socio-histórica, de manera entrelazada con las acciones de la humanidad en todo el proceso de civilización. En este sentido, podemos decir que el ser humano (SH) no es visto aquí como un ser aislado en la naturaleza, ni en sus prácticas, sino como un componente del sistema complejo que es nuestro universo, con sus reglas y funciones sistémicas, ya sean naturales o culturales.

Con la suposición de que el comportamiento no siempre coincide con la percepción de la conciencia, esta investigación se desarrolló con el objetivo de evaluar el conocimiento y el comportamiento ambiental durante las prácticas de ocio en la naturaleza, de acuerdo con las escalas y los instrumentos que se presentan a continuación. Este estudio también fue motivado por el discurso recurrente que las prácticas corporales en la naturaleza - especialmente durante el tiempo de ocio, suponen un regreso del SH a una naturaleza diferente de los entornos urbanos llenos de flora, fauna y formaciones geográficas no afectada por su acción. Este discurso hegemónico indica que sus practicantes han avanzado en su conciencia ambiental y que su comportamiento refleja la misma.

En este sentido nos preguntamos si se corresponde con la realidad, es decir, si la conciencia y el comportamiento "van de la mano" durante estas prácticas.

\section{Ámbito de la investigación}

Gran parte de las playas de Río Araguaia son formaciones no permanentes que surgen todos los años durante la estación seca, cuando el nivel del río es significativamente más bajo y se forman "islas" de arena. Son en estas formaciones temporales que ocurre una "invasión" de turistas durante las vacaciones escolares de julio. Estos turistas generalmente se propagan a través de los campamentos, llevando con ellos todo tipo de enseres imaginables: a partir del material más básico para "playa" pasando por el equipo de pesca, hasta campamentos (privado /sectoriales o familiares) los más simples a los más sofisticados, que cuentan con todas las comodidades de un hogar urbano, tales como nevera, congelador, TV, equipos de música, ventiladores, aparatos de cocina, etc. Por no hablar de la gran cantidad de barcos (y las consecuencias obvias de la misma) de todos los tamaños que pasean por el río las 24 horas del día

\section{2.- METODOLOGÍA}

Esta investigación se inscribe dentro de la investigación cualitativa - diseñada para analizar una realidad sin que sus resultados tengan que ser generalizados. En este tipo de investigación los datos más relevantes son las relaciones entre los sujetos y sus conexiones con el medio ambiente. La investigación que aquí se presenta se centró principalmente en la aplicación de un cuestionario (Escala) y en las observaciones de campo, que se llevaron a cabo durante tres días (viernes, sábado y domingo), en las islas del Rio Araguaia en sus márgenes cercanas de la ciudad de Aruanã/GO; fue realizado por tres profesores y cuatro estudiantes miembros de la investigación. Es de destacar que en estos días coincidieron con el fin de semana más ocupados en la región (tercer fin de semana de julio), según los informes de los residentes y comerciantes de la ciudad.

\section{Población y muestra}

El diseño de la muestra no siguió una determinación probabilística, sino que fuer con conveniencia. El número de cuestionarios obtenido dependió de la capacidad que el equipo de 
investigación tuvo para realizar la recolección, contabilizando un total de 235 entrevistados. Los datos del perfil de los encuestados se encuentran a continuación.

\section{Instrumento}

Para recopilar y evaluar los niveles de la conciencia ambiental y el comportamiento de los sujetos se utilizó un cuestionario, llamado por nosotros "Escala del Comportamiento Responsable", siendo una adaptación del instrumento de Baena e Inácio (2013).

En términos estadísticos, se calculó la media $(M)$ y desviación estándar $(S D)$. Con otros aspectos como las pruebas de normalidad, índices de asimetría, $T$ de Student, si determinaron y confirmaron la idoneidad del instrumento. Para tratamiento estadístico de los datos se utilizó del software SPSS versión 17.0 para Windows.

Además de aspectos como la preocupación ambiental, conciencia ambiental, conducta ecológica y sostenible, actitud, entre otros, el instrumento se amplió con preguntas acerca de las características sociales, económicas, culturales y escolares de los sujetos.

Por último, el cuestionario constaba de 18 preguntas, las cuales contenían varias subcuestiones totalizando 58 opciones de respuesta.

\section{3.- RESULTADOS}

\section{Características de los sujetos}

Sobre sus edades, 159 eran adultos, 65 jóvenes y 6 mayores, con un total de 5 personas que no contestaron a esta cuestión. Otras características, como nivel escolar, participación ciudadana, hábitos de lectura, etc., no serán presentados en este trabajo debido al límite de extensión, pero se podrán ver en otras futuras publicaciones.

\section{Conciencia y conducta}

En este trabajo, nos atendremos a las cuestiones 17 y 18 de la Escala, porque son las más estrechamente relacionados están con el fin de evaluar si hay o no una correspondencia directa entre la conciencia y la conducta o si existen diferencias/contradicciones entre estas dos variables.

Las preguntas 17.1 y 17.2, preguntaban sobre el comportamiento de los individuos cuando existe cierta presencia de fauna en el área visitada. Se observó que unos 59,15\% presentan una actitud de no acosar a los animales que encuentran, así como a sus nidos o construcciones de reproducción (64,26\%). Estos datos sugieren que los sujetos entienden la necesidad de no interferir en los entornos en los que los sistemas adonde se desarrollan sus prácticas corporales de aventura (o no), aspecto importante de la conciencia ambiental. Sin embargo, cuando no hay presencia física visible de la fauna, este comportamiento cambia. Sólo 96 sujetos (40,85\%) afirman no hacer ruido (cuestión 17.3) y más del 14\% reportaron ser indiferentes a ella.

Una primera contradicción entre la conciencia y el comportamiento ha sido encontrado aquí: aunque la gran mayoría dice que no moleste a los animales que encuentra, a otro tanto no les importa hacer ruidos o recoger elementos del espacio cuando no perciben la presencia de ciertos animales. Se sabe que los ruidos extraños hacen que el animal se mueva huyendo de los mismos, aunque no estén en ese momento mirando a nadie. Del mismo modo, recoger desperdicios del espacio contradice todas las directrices sobre el comportamiento de los SH en áreas más "naturales".

Las cuestiones 17.5, 17.6 y 17.7 se refieren a la producción y encaminamiento adecuado de la basura producida durante la práctica en la naturaleza. Se encontró que 151 sujetos (64,3\%) afirman no dejar su basura en el medio natural. Aún así, sólo 108 (46\%) de ellos manifiestan que recogen la basura que encuentran (17.6).

El aspecto crucial de estas dos cuestiones es que hay un comportamiento distinto cuando el sujeto produce la basura y cuando este mismo sujeto encuentra residuos producidos por otros. Parece que hay un entendimiento de que cada uno debe ser responsable sólo de sus propias acciones (individualismo); de esta manera la basura que se encuentra en la ruta de acceso simplemente puede ser ignorada. 
Sin embargo, este es un tema delicado, porque se puede encontrar tal cantidad de residuos que no puede ser recogido por otro individuo, o incluso que una persona tenga que abandonar su actividad debido a la cantidad de basura encontrada.

El ítem 17.7 se preguntaba si los sujetos llamaban la atención a las personas que dejaban la basura en el espacio, y sólo el 26\% respondió positivamente. En este tema, la posibilidad de conflicto entre los que dejan la basura y lo que llaman la atención es significativa. Se infiere en que es más difícil presentar un comportamiento ambiental adecuado en situaciones donde esto puede llevar a la confrontación.

Volviendo a utilizar las relaciones estadísticas, se encontró que de los sujetos que dicen que no acosan a la vida salvaje, solo una fracción $(77,7 \%)$ dice que no recoge la basura que se encuentra en su camino. Es decir, el comportamiento medioambiental flota de acuerdo con la situación vivida; aspectos que constituyen la conciencia y el comportamiento pueden presentar desconexiones en función del contexto.

Los ítems 17.10 y 17.13 se refieren al desplazamiento para la práctica en la naturaleza y cuando uno está en el lugar de práctica. Sobre el interés de preservar los senderos naturales (17.10), sólo 116 sujetos $(49,4 \%)$ fueron sensibles a estos senderos. Este dato es muy importante debido a la importancia que las vías naturales tienen el equilibrio del ecosistema local. Si no hay preocupación con esto, se puede ocasionar en el futuro gran cantidad de molestias.

En relación a los desplazamientos para llegar a los espacios de las prácticas (17.3), el 38,3\% de los sujetos afirman estar interesados en el tipo de transporte que utilizan. Aquí se observó otro conflicto: parte significativa de los sujetos se preocupan con los senderos naturales que encuentran, pero no se preocupan con su propio desplazamiento hasta allí - otra vez se puede ver que el contacto más directo con la naturaleza o medio afecta el comportamiento. Por lo tanto, cuando los sujetos están en el entorno natural, la preocupación ambiental se amplía; al contrario, si no están en la naturaleza, se diluye tal preocupación. Este hallazgo se alinea con el pensamiento de Milton Santos, en la interferencia de espacio en el comportamiento humano.

Pasamos ahora a la pregunta 18, que se refiere más a la conciencia, donde las respuestas de los sujetos se encuentran más en el nivel cognitivo que de la acción misma. En la cuestión 18.1 se preguntaba si los sujetos estarían dispuestos a hacer sacrificios personales para reducir el índice de contaminación del medio ambiente, aunque los resultados iniciales no fueron evidentes. Se encontró que más del $74 \%$ de los sujetos estaban de acuerdo o totalmente de acuerdo con esto. Pero cuando llegamos a las cuestiones 18.2, 18.3 y 18.4, que están relacionadas con las comodidades cotidianas de los sujetos, tal conciencia se desconecta del comportamiento. En la pregunta 18.2, sólo 65 (27\%) de los sujetos dijeron que iban a dejar sus coches en casa si el transporte público fuera eficiente, mientras que 45 (19,1\%) de ellos no estaba de acuerdo en cambiar sus coches por el transporte público. Una vez más con la ayuda de la estadística, se infiere que de aquellos que respondieron positivamente a hacer sacrificios personales para el MA, sólo el 37 \% de ellos cambiarían su medio de transporte.

En la cuestión 18.3 se preguntaba si los sujetos darían una parte de su tiempo o una donación en efectivo a una organización ambiental. Aquí, sólo 72 (30,6 \%) sujetos acordaron, presentando una vez más un conflicto con los 116 que declararan su voluntad de auto-sacrificio. A su vez, el ítem 18.4 cuestionó específicamente si los sujetos donarían 30 Reales brasileños ${ }^{2}$ por año a las organizaciones ambientales. El resultado mostró que sólo 67 de ellos (28,5\%) lo harían, de nuevo en contraste con los 116 concordantes con sacrificios personales.

El análisis de las cuestiones de la Escala, de una manera global, apunta que el 90,8\% de los sujetos tiene una conciencia ambiental importante, siendo significativas sus respuestas a las preguntas sobre el cuidado del medio ambiente. Finalmente, ha sido posible inferir - estadísticamente, que unos $90 \%$ de los sujetos conscientes será el mismo tanto por ciento que presentará un comportamiento responsable.

\footnotetext{
${ }^{2}$ Cerca de $10 €$
} 


\section{4.- CONCLUSIONES}

Este estudio tuvo como objetivo evaluar los niveles de conciencia y el comportamiento ambiental de los sujetos frecuentadores de un cierto medio natural para sus vacaciones de julio.

La investigación ha demostrado que hubo una correlación bastante importante entre conciencia y comportamiento de los sujetos. Este hallazgo es diferente de lo obtenido en otras investigaciones (Baena y Inácio, 2013), en la cual se encontró una descompensación entre las dos variables. Todavía, hay que analizar los datos recopilados desde la observación de campo, apuntados en un diario y registrados en las fotos.

La observación de cada día nos mostró una realidad distinta de la que nos apuntan los resultados arriba presentados: dependiendo del tipo de campamiento - asociativo, familiar, corporativo o de un día, lo que miramos era que en los campamientos asociativos había una orden, una organización que llevaba a sus integrantes a presentar un comportamiento bastante ejemplar; a la medida que pasamos a los campamientos corporativos, familiares y los de un día, las cosas se cambian respectivamente "del cielo al infierno". La investigación ha dejado claro unos comportamientos absurdos especialmente en los campamientos de un día: basura en todas partes, botellas de vidrio y aluminio tiradas en las playas y en el río, lavado de ropas y vajillas con detergentes químicos en el propio río, barbacoas una al lado de otra (centenares) produciendo mucho humo y una mezcla de olores completamente distinta al medio. A todo esto hay que añadirle la existencia de muchísimos campamentos con su propio equipo de música (a un alto volumen), lo que constituye un ruido absurdo las 24 horas del día con respecto al lugar en el que se encuentran.

Se halló además, en el sentido contrario, algunos agentes de órganos gubernamentales con campañas de concientización, bomberos y policías implicados en cambiar tal orden. Pero esto constituía una tarea ardua y muy difícil, especialmente por el gran número de turistas y el bajísimo personal a trabajar.

Sobre la correlación final entre conciencia y comportamiento, entre los investigadores se ha quedado una sensación muy palpable de que los sujetos respondían de manera distinta a lo que realmente piensan, sea para justificar respuestas ambientalmente correctas, sea porque - de hecho, piensan de una manera y se comportan de otra, sin la debida percepción de sus propias contradicciones; pero esto es una conclusión basada en impresiones, no en los datos concretos.

Si fuera esta una investigación basada en la Fenomenología, entonces las impresiones tendrían validad para los análisis. Es importante resaltar que las cuestiones que no se tratan en este texto (de la 01 hasta la 16) nos proporcionan docenas de datos muy importantes, que serán objeto de publicación en otros medios. Categorías como el género, la edad, la condición social, la participación ciudadana, los hábitos diarios, etc., proporcionarán información muy interesante, como por ejemplo, que la conciencia ambiental de las mujeres era más positiva que de los hombres. Importa también recordar que este estudio se llevó a cabo en Brasil, en un contexto muy específico, con un grupo muy peculiar de sujetos. Por lo tanto, se sugiere que se puede replicar en otros contextos, buscando identificar aspectos particulares, sin la cual la producción de conocimiento se vacía de significado y relevancia.

Por último, queda por hacer hincapié en que entendemos el papel del ocio como absolutamente significativo en la (re)construcción de valores y actitudes para una vida ética, justa y armoniosa, en todas las parcelas y tesitura social en el que operamos

\section{5.- REFERENCIAS}

Corraliza, J. A. y Martín, R. (2000). Estilos de vida, actitudes y comportamientos ambientales. Medio Ambiente y Comportamiento Humano, 1(1), 31-56.

Inácio, H.L.D. y Baena, A. (2013). Relação entre consciência e comportamento ambientais em práticas de lazer na natureza. En: XVIII Conbrace y V Conice (Actas), Brasília. CBCE.

Rodríguez, M., Boyes, E., y Stanisstreet, M. (2010). Spanish Secondary students` willingness to undertake specific actions to combat global warming: Can environmental education help? Psychology, 1(1), 1-17. 